Wilfrid Laurier University

Scholars Commons @ Laurier

Physics and Computer Science Faculty

Publications

Physics and Computer Science

1996

\title{
Chaos and Thermal Noise in a Josephson Junction Coupled to a Resonant Tank
}

James A. Blackburn

Wilfrid Laurier University, jabjabjab@cogeco.ca

H.J.T. Smith

University of Waterloo

Niels Grønbech-Jensen

Los Alamos National Laboratory

Follow this and additional works at: https://scholars.wlu.ca/phys_faculty

\section{Recommended Citation}

Blackburn, James A.; Smith, H.J.T.; and Grønbech-Jensen, Niels, "Chaos and Thermal Noise in a Josephson Junction Coupled to a Resonant Tank" (1996). Physics and Computer Science Faculty Publications. 28.

https://scholars.wlu.ca/phys_faculty/28

This Article is brought to you for free and open access by the Physics and Computer Science at Scholars Commons @ Laurier. It has been accepted for inclusion in Physics and Computer Science Faculty Publications by an authorized administrator of Scholars Commons @ Laurier. For more information, please contact scholarscommons@wlu.ca. 


\title{
Chaos and thermal noise in a Josephson junction coupled to a resonant tank
}

\author{
James A. Blackburn \\ Department of Physics and Computing, Wilfrid Laurier University, Waterloo, Ontario, Canada N2L $3 C 5$ \\ H. J. T. Smith \\ Department of Physics, University of Waterloo, Waterloo, Ontario, Canada N2L 3G1 \\ Niels Grønbech-Jensen \\ Theoretical Division, Los Alamos National Laboratory, Los Alamos, New Mexico 87545 \\ (Received 20 December 1995)
}

\begin{abstract}
Selected dynamical modes are investigated for the autonomous system formed from a dc biased Josephson junction which is resistively coupled to a resonant tank. A hysteretic zone in the current-voltage characteristic is shown to result from coexisting chaotic and periodic states. The detailed features of these states, including the geometrical structure of the attractors and their basins of attraction, as well as thermally induced transitions between them, are explored. [S0163-1829(96)08021-6]
\end{abstract}

\section{INTRODUCTION}

Since the seminal work on chaos in Josephson junctions by Huberman et al., ${ }^{1}$ it has been realized that superconducting weak links may exhibit chaotic dynamical behavior. The most commonly studied configuration has been that of a capacitive Josephson junction under the influence of ac current excitation. This nonautonomous system, governed as it is by a second-order differential equation with an external harmonic forcing function and presumed to model a junction under the influence of microwave irradiation, displays all of the rich dynamics which might be expected of a driven nonlinear device. ${ }^{2}$

Jensen et al. $^{3}$ showed that a capacitive junction with only dc bias, but loaded by a series or parallel resonant tank, possesses chaotic modes. Along similar lines, Pedersen and Davidson $^{4}$ noted that chaos occurs when a dc biased capacitive junction is coupled to a simple $R-L$ load. Without ac bias, these cases are both autonomous, but with the inclusion of inductance, the equations are of third order. The essential ingredient in these cases is, therefore, the inductance.

As prospective components in superconducting electronics, ${ }^{5}$ Josephson devices of small physical dimensions are clearly preferred. Small junctions have correspondingly small, or even negligible, associated self-capacitance. In this limit neither the nonautonomous isolated ac driven junction nor the autonomous inductively loaded dc biased junction can become chaotic, since in both cases the equations are only of second order. However the possibility of chaos returns when two or more zero-capacitance junctions are coupled in arrays. ${ }^{6}$

Another interesting and physically realistic arrangement which can give rise to chaos consists of a dc biased Josephson junction of negligible capacitance (highly overdamped) which is coupled to a tank circuit containing both inductance and capacitance. This particular system is the subject of our study. It is conceptually appealing because it is autonomous and so is free of the ambiguity which ac forcing inevitably introduces - that of relating simulation ac amplitudes to experimentally applied rf fields. Although the results to be presented here were obtained entirely from numerical calculations, all the parameters are in principal relatively easy to quantify in an experimental setup.

This paper addresses two principal issues. The first is concerned with the appearance of chaotic states in the parameter space of the system. As is typical in such investigations, the parameter values chosen for detailed dynamical simulations are not in any way special except in the sense that they lead to significant and interesting modes. Of paramount interest is a range of dc bias current within which chaotic and periodic states coexist. The second topic concerns the response of the system to the presence of thermal noise. Not surprisingly, thermally activated transitions are found to occur between coexisting states; the rate of such transitions will be used to infer effective energy barriers.

\section{JOSEPHSON JUNCTION AND TANK}

Figure 1 illustrates a Josephson junction (the bowtie labelled $\sin \phi$ ) coupled via resistor $r$ to a tank made up of the parallel combination of inductor $L$ and capacitor $C$. Both the coupling resistor and the junction shunt $R_{J}$ are shown with Johnson noise sources. The following equation is immedi-

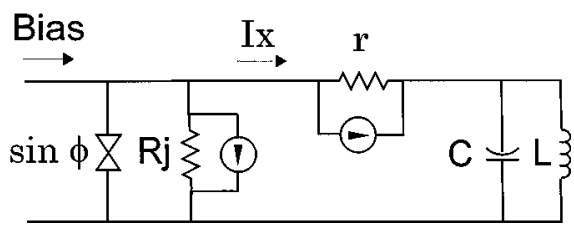

FIG. 1. Josephson junction resistively coupled to a resonant tank comprised of a parallel inductor and capacitor. Noise currents shunt the two resistors. 
ately evident from Kirchhoff's laws:

$$
I_{B}=I_{C} \sin \phi+\frac{V_{J}}{R_{J}}+I_{N}^{J}+I_{X},
$$

where $I_{B}$ is the external dc bias current, $I_{C}$ is the junction critical current, $\phi$ is the junction phase parameter, and $V_{J}$ and $R_{J}$ are the junction voltage and shunt resistance, respectively. The noise current associated with the junction normal resistance is denoted $I_{N}^{J}$, while $I_{X}$ represents the current flowing into the coupling resistor. But

$$
I_{X}=I_{N}^{r}+\frac{\left(V_{J}-V_{T}\right)}{r}
$$

in which $V_{T}$ signifies the tank voltage (across $C$ or $L$ ) and $I_{N}^{r}$ is the noise source shunting the coupling resistor. The junction voltage and phase are connected by the Josephson relation

$$
\frac{d \phi}{d t}=\frac{2 e V_{J}}{\hbar}
$$

In what is to follow, it will be useful to employ the dimensionless parameters:

$$
\alpha=\frac{\hbar}{2 e} \frac{1}{\left[L I_{C}\right]} \quad \beta=\frac{\hbar}{2 e} \frac{1}{\left[C I_{C} R_{J}^{2}\right]} \quad \gamma=\frac{r}{R_{J}} .
$$

Since $\hbar / 2 e I_{C}$ is an effective junction inductance ${ }^{7} L_{J}, \alpha$ is the ratio of Josephson inductance to tank inductance. Similarly, $\beta$ is the ratio of two time constants, $L_{J} / R_{J}$ and $R_{J} C$. Normalizing current as $i=I / I_{C}$, voltage as $v=V / I_{C} R_{J}$, and time as $\tau=t\left[2 e I_{C} R_{J} / \hbar\right]$, and combining Eqs. (1 ) and (2),

$$
\dot{\phi}=\frac{\gamma}{\gamma+1}\left[i_{B}-\sin \phi+\frac{v_{T}}{\gamma}-i_{N}^{J}-i_{N}^{r}\right],
$$

where the overdot signifies a derivative in normalized time. The law of induction $V_{T}=L\left(d I_{L} / d t\right)$ becomes

$$
i_{L}=\alpha\left[v_{T}\right]
$$

Finally, beginning with an expression for the capacitor current, $I_{\text {cap }}=C\left(d V_{T} / d t\right)$ and using $I_{\text {cap }}=I_{X}-I_{L}$, we have

$$
\dot{v}_{T}=\frac{\beta}{1+\gamma}\left[i_{B}-\sin \phi-v_{T}-i_{N}^{J}+\gamma i_{N}^{r}\right]-\beta i_{L} .
$$

The noise current source associated with the finite operating temperature for resistor $R_{J}$ is given by the standard expression $^{8-11}$

$$
I_{N}^{J}=\sqrt{\frac{2 k T}{R_{J} \delta t}} N(0,1) .
$$

$N(0,1)$ is a random number with Gaussian distribution, zero mean, and variance $1 ; \delta t$ is the time grid for the numerical integrations and on which the random numbers are updated. Using the previously defined dimensionless time scale and employing the Josephson-junction coupling energy $E_{J}=\hbar I_{C} / 2 e$, this becomes

$$
i_{N}^{J}=\sigma N(0,1)
$$

with

$$
\sigma=\sqrt{\frac{2 k T}{E_{J}} \frac{1}{\delta \tau}}
$$

It is easily seen that

$$
i_{N}^{r}=i_{N}^{J} \gamma^{-1 / 2}
$$

Equations (4),(5), and (6) can then be expressed in final form as

$$
\begin{gathered}
\dot{\phi}=\frac{\gamma}{\gamma+1}\left[i_{B}-\sin \phi+\frac{v_{T}}{\gamma}-\sigma\left(N_{1}+\frac{N_{2}}{\sqrt{\gamma}}\right)\right], \\
\dot{i}_{L}=\alpha\left[v_{T}\right], \\
\dot{v}_{T}=\frac{\beta}{1+\gamma}\left[i_{B}-\sin \phi-v_{T}-\sigma\left(N_{1}-\sqrt{\gamma} N_{2}\right)\right]-\beta i_{L},
\end{gathered}
$$

with $N_{1}$ and $N_{2}$ denoting independent random numbers of the form $N(0,1)$. Hence the coordinates of the autonomous system are the junction phase, inductor current, and tank voltage, $\left(\phi, i_{L}, v_{T}\right)$, and its evolution in time can be visualized in the form of trajectories in this three-dimensional space. Apart from initial conditions, numerical values for the three dimensionless parameters $(\alpha, \beta, \gamma)$ must be specified as well as an amplitude for the noise parameter $\sigma$ (equivalent to setting the temperature).

In a graduate thesis by Macki, ${ }^{12}$ computed current-voltage characteristics for the junction/tank system with the parameter set $\left[\alpha=0.07 ; \beta=\frac{2}{7} ; \gamma=\frac{3}{7}\right]$ contained a number of interesting features, including a hysteresis loop located in the vicinity of $i_{B}=1.9$. This same set was adopted for the present study.

It is worth taking note of the time scales in this problem. The tank circuit by itself has the well-known resonant frequency $\omega_{0}=[L C]^{-(1 / 2)}$; its corresponding period in normalized time units can be shown to be $P_{T}=2 \pi[\alpha \beta]^{-(1 / 2)}$. By comparison, an isolated Josephson junction with a bias of say twice the critical current would oscillate at approximately $\omega_{J} \approx 2\left[2 e I_{C} R_{J} / \hbar\right]$, which has a corresponding period, in normalized time units, of $P_{J}=\pi$.

Numerical solutions of the system of coupled first-order differential equations (9), (10), and (11)] were computed using a fourth-order Runge Kutta routine with fixed step size. For the parameter set just mentioned, the characteristic periods for tank and junction oscillations would be $P_{T}=44.43$ and $P_{J}=3.14$. Hence the shorter time scale of $P_{J}$ must be considered when choosing the step size for numerical integrations. For the results presented here, a $\delta \tau$ of about $0.001 P_{J}$ was used, although a coarser grid would likely have been acceptable.

\section{DYNAMICS: NOISELESS CASE $(\sigma=0)$}

Of central importance to the material in this and the following sections will be the representation of Poincare plots. Since the junction phase enters Eqs. (9), (10), and (11) only 


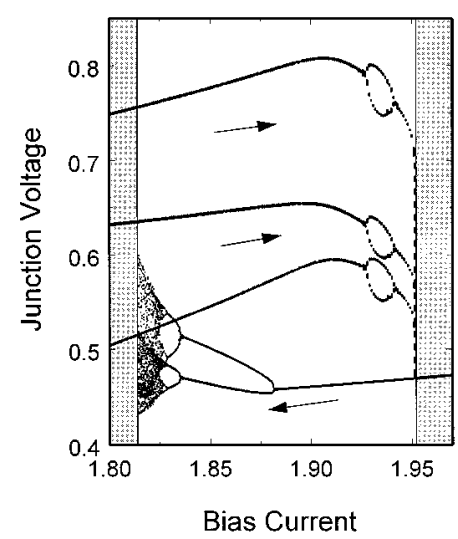

FIG. 2. Bifurcation diagram illustrating ascending and descending branches in the hysteresis loop. Beyond the hysteresis (shaded regions) the mode is either period-3 or period-1.

as $\sin \phi, \phi \pm m(2 \pi)$ will also always be a solution and the phase variable can (if it is useful to do so) then be restricted to the range $-\pi$ to $\pi$. Because this is an autonomous system, obviously there is no external "strobe." Instead, we choose to sample the coordinates $i_{L}$ and $v_{T}$ every time the phase variable $\phi$ reaches some preset reference value. The selection of this reference value is entirely arbitrary, although the visual appearance of the resulting Poincare section depends upon the choice made. Unless specified otherwise, a reference phase of $\pi$ was used.

The hysteresis loop in the current-voltage characteristic for this system, which was alluded to in the previous section, spans the range $1.8139 \leqslant i_{B} \leqslant 1.9520$. The upper and lower branches of this loop arise from distinct dynamical modes which clearly coexist at any selected bias current within the domain of the hysteresis. Such coexistence is clearly evident in the bifurcation diagram shown in Fig. 2. For each setting of bias current, values of junction voltage $(\dot{\phi})$ (sampled at $\phi=\pi$ ) are plotted. Clearly, below the onset of the hysteresis at $i_{B}=1.8139$ only a period-3 solution prevails. Above the terminating point of the loop at $i_{B}=1.9520$ only a period-1 solution exists. When the loop is entered from below and the bias is slowly incremented, the period-3 solution prevails, with the exception of the brief doubled behavior in the miniloops, until the end point is reached. Conversely, if the loop is entered from above and the bias is slowly decremented, then a period doubling sequence occurs leading to chaos, before the abrupt return to period-3.

This study focusses on the region of bias current just above 1.8139 because here is found an interesting coexistence of a chaotic state with a periodic state. Therefore we now fix the bias current at $i_{B}=1.8150$. As remarked above, the period-3 state can be reached by ramping the bias up to 1.8150 from below the loop, while the chaotic state can be reached by ramping the bias down from above the loop. Suppose, instead, that the numerical solution of system (9), (10), (11) is computed directly from a specific initial condition, $\left[\phi(\tau=0) ; i_{L}(\tau=0) ; v_{T}(\tau=0)\right]$. In such a case, the system ultimately will reach either the chaotic state, or the period-3 state. The domain of starting values leading to a given attractor is its basin of attraction.

Figure 3 is a composite plot in the $i_{L}-v_{T}$ plane containing both attractors and basins (the reversed direction on the

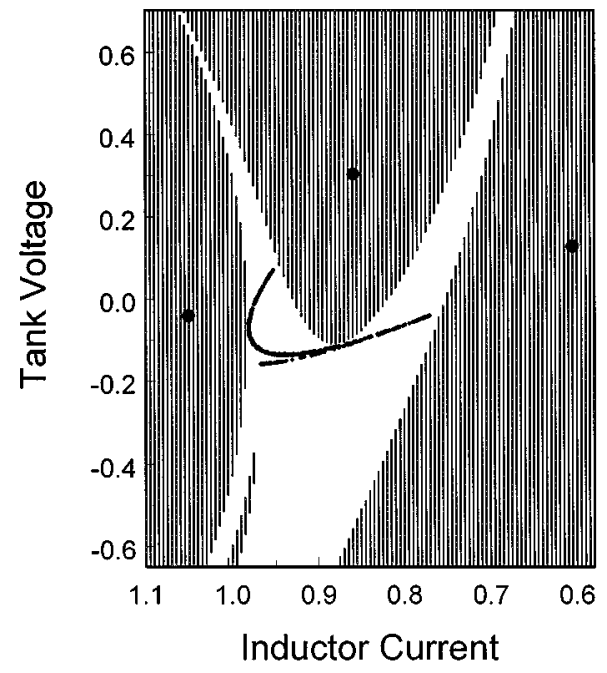

FIG. 3. Poincaré section (fishhook) and period-3 attractor (black dots) for a reference phase $\phi=\pi$. The shaded region is the basin of attraction for the period-3 state; the white region is the basin of attraction for the chaotic state.

$i_{L}$ axis has been chosen for visual consistency with some figures to follow). One of the three initial conditions is preset, namely $\phi=\pi$. The period-3 state appears as three dots. The chaotic state produces the fishhook. When magnified, this fishhook has a finite thickness and is fractal, as usual for a strange attractor. The white regions of the figure represent the basin of attraction of the chaotic state; the black regions are the basin for the period-3 orbit. Note the near contact of the strange attractor with the basin boundary in two places, while the period-3 attractor (black dots) is situated deep within its own basin. As we shall see, this implies that it will be much easier to force transitions out of chaos than the reverse. Figure 4 is constructed in the same manner as Fig. 3, except that the reference phase here is $\phi=0$.

The Poincaré sections in Figs. 3 and 4 (fishhooks) are produced by just two particular choices for the definition of the strobing event, $\phi=0$ or $\pi$. In other words, the complete

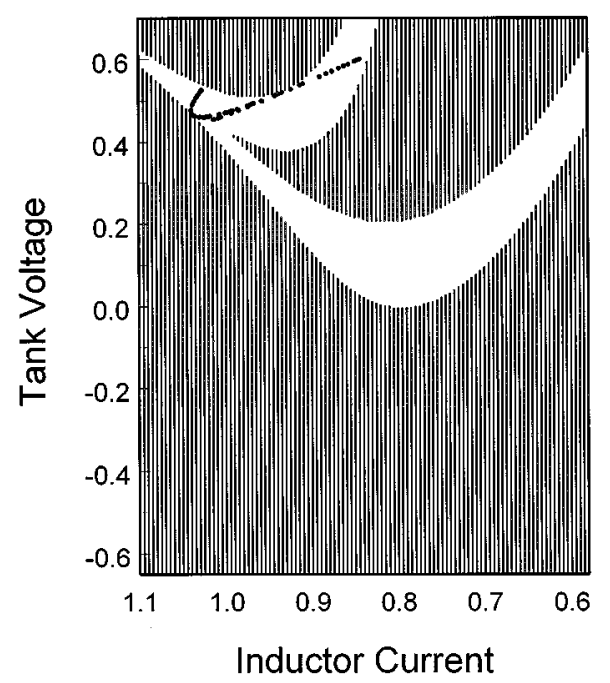

FIG. 4. Same as the Fig. 3, but taken at a reference phase $\phi=0$. 


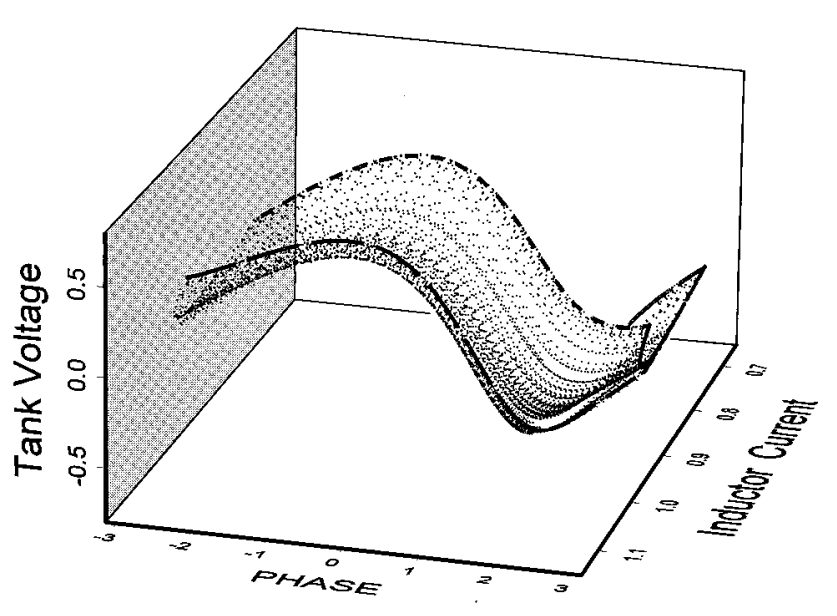

FIG. 5. Complete strange attractor in $\left[i_{L}, v_{T}, \phi\right]$ space for $i_{B}=1.815$. The surface is a fractal Möbius band, twisting in the vicinity of $\phi=2$ radians.

strange attractor in the space $\left[\phi, i_{L}, v_{T}\right]$ is only hinted at by each of these sections. The entire strange attractor is illustrated in Fig. 5. The end caps at $\phi= \pm \pi$ are just the fishhooks in Fig. 3; Fig. 4 corresponds to a slice at $\phi=0$. This surface is, in fact, a fractal Möbius ribbon in which the twist occurs in the neighborhood of $\phi=2$ radians. A line along the back edge advances to the front while the leading edge rolls under and moves to the rear.

The period-3 attractor is shown in Fig. 6 where it is represented by a trajectory which advances steadily in the phase variable. This curve completes a full cycle after any phase advance of $3 \times 2 \pi$, a property which can be discerned in the figure by noting that the starting point on the $\left[v_{T}, i_{L},-\pi\right]$ plane is the same as the finishing point on the $\left[v_{T}, i_{L}, 5 \pi\right]$ plane. This figure also includes three cycles of the strange attractor joined end on end. The period- 3 trajectory can be seen to wrap itself once around the Möbius washboard in the course of the net phase advance of $6 \pi$. A view of this same period-3 orbit looking down the phase axis (or, equivalently, projected onto the $i_{L}-v_{T}$ plane) is shown in Fig. 7. Also plotted is the projection of the strange attractor on this same plane, and in this view the chaotic nature of the orbit is quite apparent.

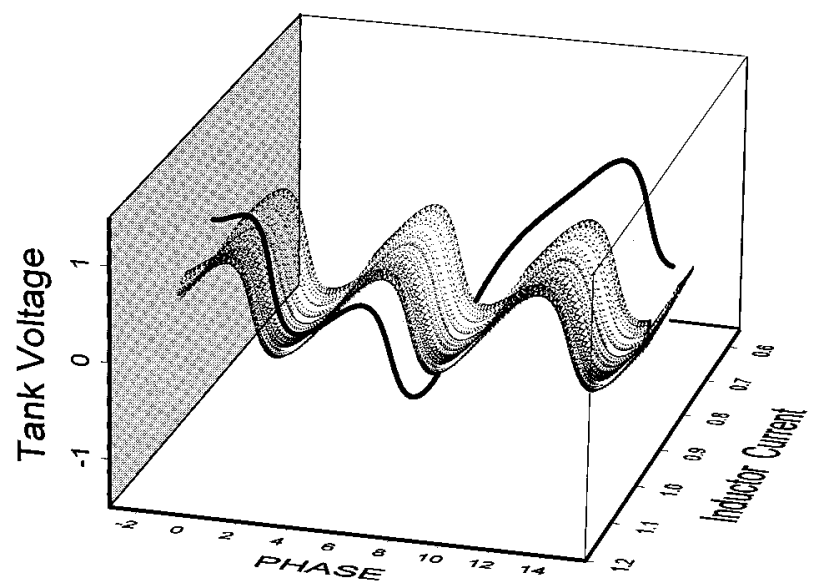

FIG. 6. Three cycles of the strange attractor, and a complete coexisting period-3 orbit for $i_{B}=1.815$.

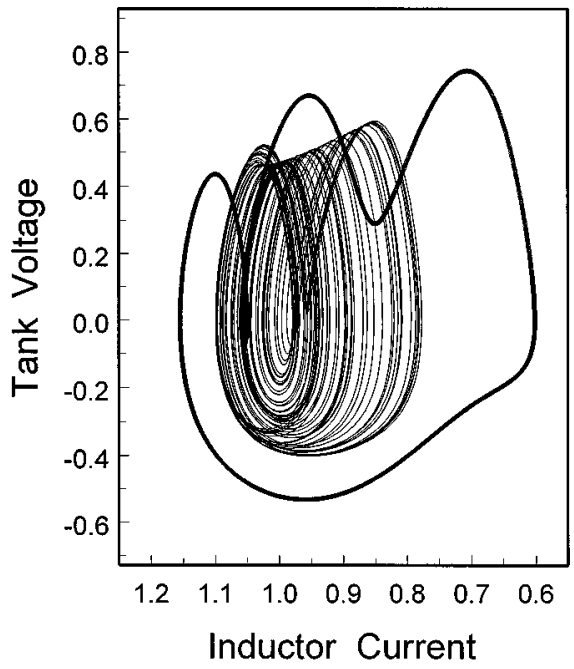

FIG. 7. Strange attractor and period-3 orbits projected onto the $i_{L}-v_{T}$ plane.

\section{DYNAMICS: FINITE TEMPERATURE $(\sigma>0)$}

The addition of thermal (or indeed other forms of) energy to this system will perturb whichever of the two coexisting states may be occupied at a given moment. Intuitively, it can be appreciated that given sufficient thermal noise and after an adequate amount of time, excitation from one attractor to the other can take place. Flipping back and forth between attractors is also possible, although the directional transfer rates may be extremely unequal.

The issue of thermally driven escape from attractors is of some practical significance, noise being ubiquitous. It is also an appealing topic because it links classical stochastic theory with modern nonlinear dynamics. Furthermore, the dependence of mean lifetime on noise amplitude serves as a probe of the effective energy barriers of the system.

Both ac driven Josephson junctions, ${ }^{10,13}$ and logistic ${ }^{14}$ as well as quadratic ${ }^{15}$ maps have served as model systems. As discussed by Kautz ${ }^{10}$ and Beale,${ }^{14}$ the mean escape time for thermal excitation from an attractor is governed by an expression of the form

$$
\left\langle\tau_{\text {esc }}\right\rangle=\tau_{0} \exp \left[\frac{E_{0}}{k T}\right],
$$

where $E_{0}$ is the depth of the effective potential well containing the system. Thus a plot of the natural log of mean escape time versus reciprocal noise temperature should be a straight line of slope $E_{0}$ (see Kautz ${ }^{10}$ Fig. 3, Beale ${ }^{14}$ Fig. 2, and Grassberger ${ }^{15}$ Fig. 2). We follow a similar procedure here to evaluate the barrier height for noise-driven transitions out of chaos. Note that $\sigma^{2} \delta \tau=2 k T / E_{J}$ and so the slope of a graph of $\ln \left(\left\langle\tau_{\text {esc }}\right\rangle\right)$ versus $2\left[\sigma^{2} \delta \tau\right]^{-1}$ would be

$$
\text { slope }=\epsilon,
$$

with $\epsilon \equiv E_{0} / E_{J}$ being the normalized barrier height.

For selected noise amplitudes, $\sigma$, the mean escape time was determined as follows. The numerical simulation of the system equations (9), (10), and (11) was started on the strange attractor and followed until it fell within small target zones surrounding each of the period-3 attractor points 


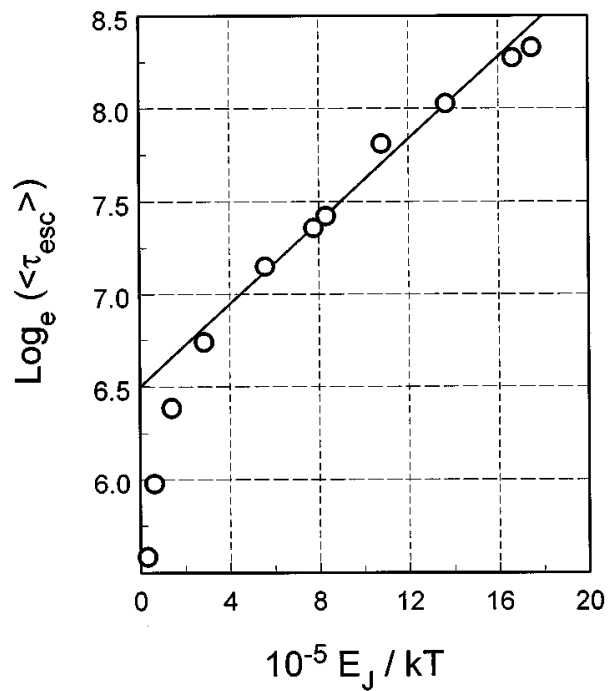

FIG. 8. Natural logarithm of the mean lifetime on the chaotic attractor prior to a thermally induced escape, as a function of reciprocal temperature. The slope of the line is $0.11 \times 10^{-5}$ (a dimensionless quantity).

marked in Fig. 3. Then the previous initial conditions were used in a noiseless simulation which proceeded until the next point in the Poincare section was reached. This point then served as a fresh initial condition for a new escape simulation (with noise once again). Because each successive point on a strange attractor is not correlated with previous values, the set of escape times found in this way yields a meaningful average. In the present study, 200 trials were carried out for each selected $\sigma$. The results are displayed in Fig. 8. The deviation from linearity at small abscissae is not unexpected since the thermal activation model is thought to apply only at low temperatures. As the temperature rises (moving to the left) escape routes other than the minimum energy one characterized by $\epsilon$ become possible, and the mean lifetime begins to decline rapidly. From the slope of $0.11 \times 10^{-5}$ one obtains a barrier height of $\epsilon=1.1 \times 10^{-6}$. This number illustrates the very small depth of the effective potential well within which the chaotic state resides. Of course the particular value of $\epsilon$ will depend quite sensitively on the nearness of the bias current $i_{B}$ to the boundary value (1.8139 in this example), at which $\epsilon$ vanishes.

The reverse process in which thermal activation drives the system from the periodic attractor into chaos is also possible, but the required thermal energies are much larger. In other words the effective barrier for transitions in this direction is higher than for escapes from chaos. Similar results were found by Kautz. ${ }^{10}$ We did not carry out any systematic data generation because of the very much longer simulation runs which are necessary, although this certainly can be done in principle. One implication of these results is that relatively small amounts of thermal noise will drive the system out of a chaotic state, but probably not into one. Hence a burst of weak noise here would serve the purpose of "clearing" chaos. At higher steady noise levels, hopping back and forth between chaotic and periodic states would occur. The residency times in the two coexisting states would not be equal,

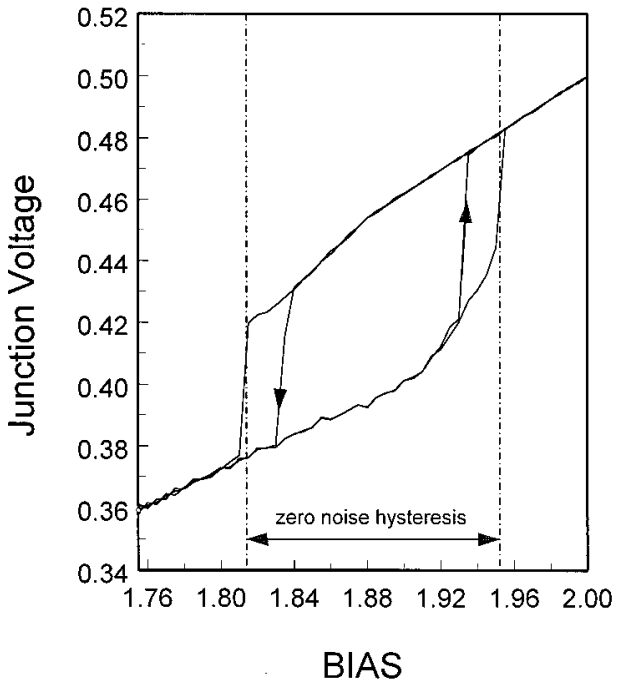

FIG. 9. Hysteresis loop in the current voltage characteristic for the junction, with and without noise. For the computational grid used here, the noise amplitude $\sigma=0.10$ corresponds to $k T / E_{J}=1.4 \times 10^{-5}$.

but their relative magnitudes would depend on the closeness of the bias $i_{B}$ to the boundary value.

Thermal noise will affect the observed hysteresis. Near the ends of the hysteresis loop, as we have just seen, the effective barrier separating coexisting states becomes quite small, at least for transitions in one of the directions. Suppose, for example, the hysteresis loop is entered from above. In the presence of noise, the lifetime on the chaotic portion of the upper branch of the loop will decrease significantly as the boundary at $i_{B}=1.8139$ is approached from the high current side. A premature switching from the upper branch to the lower one will then occur. Likewise, on the ascending period-3 branch an early transfer to the upper period-1 branch takes place before the boundary at $i_{B}=1.9520$ is reached. Higher noise temperatures will enhance this process of hysteresis loop shrinkage. These features are illustrated in Fig. 9.

The amount of shrinkage, as discussed above, and its impact on the observability of a given loop, depends of course on the precise values of circuit parameters, as well as the junction critical current and temperature. As a representative example, consider a Josephson junction of negligible capacitance with critical current $I_{C}=1 \mathrm{~mA}$. The corresponding junction coupling energy $E_{J}$ would then be about $2 \mathrm{eV}$. With the previously selected parameter values $[i=1.815, \alpha=0.07, \beta=2 / 7]$ the barrier separating chaos from period three was found to be approximately $10^{-6} E_{J}$-which is about $2 \mu \mathrm{eV}$. At a temperature of say 1 $\mathrm{mK}$ the mean thermal energy, $k T$, is of the order of $0.1 \mu \mathrm{eV}$, a figure which is at least 20 times smaller than the barrier height. Thus the chaotic state should have an adequate lifetime in the presence of such thermal noise to assure observability. If the junction had a typical resistance of $1 \Omega$, then the $\alpha, \beta$ values above would imply a tank capacitance $C=1.15 \mathrm{pF}$, a tank inductance $L=4.7 \mathrm{pH}$, and a tank resonant frequency of $70 \mathrm{GHz}$. These model component values are in fact rather close to those quoted ${ }^{16}$ for 4 - $\mu$ m-diameter 
circular $\mathrm{NbAl}_{2} \mathrm{O}_{3} \mathrm{Nb}$ Josephson junctions interconnected via superconducting microstrip lines $\left[I_{C}=365 \mu \mathrm{A}, R_{J}=3.5 \Omega\right.$, $C=0.10 \mathrm{pF}, L=7.25 \mathrm{pH}]$. We mention these similarities mainly to emphasize the plausibility of our choice of parameter values.

As a final remark, we speculate that a more extensive investigation of the system behavior likely would turn up values for $\left[i_{C}, \alpha, \beta, \gamma\right]$ for which more robust hysteresis loops exist-that is loops possessing even larger barriers against thermal activation.

\section{ACKNOWLEDGMENTS}

Financial support was provided by a grant from the Natural Sciences and Engineering Research Council of Canada. Parts of this work were performed under the auspices of the U.S. Department of Energy.
${ }^{1}$ B.A. Huberman, J.P. Crutchfield, and N.H. Packard, Appl. Phys. Lett. 37, 750 (1980).

${ }^{2}$ R.L. Kautz and R Monaco, J. Appl. Phys. 57, 875 (1985).

${ }^{3}$ H.D. Jensen, A. Larsen, and J. Mygind, Physica B 165/166, 1661 (1990).

${ }^{4}$ N.F. Pedersen and A. Davidson, in Stimulated Effects in Josephson Devices, edited by M. Russo and A. Costabile (World Scientific, Singapore, 1988).

${ }^{5}$ Superconducting Electronics, Vol. 59 of NATO Advanced Study Institute, Series F: Computer and Systems Science, edited by H. Weinstock and M. Nisenoff (Springer-Verlag, Berlin Heidelberg, 1989).

${ }^{6}$ M.A.H. Nerenberg, J.H. Baskey, and J.A. Blackburn, Phys. Rev. B 36, 8333 (1987).

${ }^{7}$ T. Van Duzer and C.W. Turner, Principles of Superconductive
Devices and Circuits (Elsevier, Amsterdam, 1981), p. 185.

${ }^{8}$ B.I. Bleaney and B. Bleaney, Electricity and Magnetism (Oxford University Press, New York, 1957) Chap. XVII.

${ }^{9}$ D.T. Gillespie, Am. J. Phys. 61, 1077 (1993).

${ }^{10}$ R.L. Kautz, Phys. Lett. A 125, 315 (1987).

${ }^{11}$ J.A. Blackburn, N. Grønbech-Jensen, and H.J.T. Smith, Phys. Rev. Lett. 74, 908 (1995).

${ }^{12}$ Michael J. Macki, MSc thesis, University of Waterloo, 1993.

${ }^{13}$ M. Iansiti, Q. Hu, R.M. Westervelt, and M. Tinkham, Phys. Rev. Lett. 55, 746 (1985).

${ }^{14}$ P.D. Beale, Phys. Rev. A 40, 3998 (1989).

${ }^{15}$ P. Grassberger, J. Phys. A 22, 3283 (1989).

${ }^{16}$ V.K. Kaplunenko, B.H. Larsen, J. Mygind, and N.F. Pedersen, J. Appl. Phys. 76, 3172 (1994). 